\title{
Over-the-counter preventive and therapeutic oral products
}

\section{Marisa Maltz(a)}

(a) $\mathrm{PhD}$, Professor of Preventive and Social Dentistry, School of Dentistry, Federal University of Rio Grande do Sul, Porto Alegre, RS, Brazil.

\author{
Corresponding author: \\ Marisa Maltz \\ Faculdade de Odontologia - UFRGS \\ Departamento de Odontologia Preventiva \\ e Social \\ Rua Ramiro Barcelos, 2492, Bom Fim \\ Porto Alegre - RS - Brazil \\ CEP: 90035-003 \\ E-mail:mmaltz@ufrgs.br
}

$\mathbf{F}_{\mathrm{o}}^{\circ}$ or most of the last century, when it came to oral care, the profession of dentistry was the only scientific and professional resource available to the individual. This reality has changed and the industry has led the way in scientific research on a vast array of over-the-counter (OTC) dental products. Nowadays, a range of products effective in treating a number of oral health diseases and meeting patients' desires is available in the marketplace. There are products for managing dental decay, tartar buildup, gingivitis, dental hypersensitivity, teeth staining, dental erosion and for a variety of other oral needs and desires. Self-medication with over the counter medicines has long been a feature of the lay health system. Due to the existence of a wide variety of OTC dental products, now the public has access to treatments previously available only through the dentist.

Considering the many choices of OTC products available in the marketplace, it is increasingly difficult to select the proper product(s). A large supply and variety of OTC dental products has prompted a great number of questions by the population and even by the general dentist.

In September 2008, the Brazilian Association for Oral Health Promotion (ABOPREV - Associação Brasileira de Odontologia de Promoção de Saúde) organized a symposium aimed to discuss various aspects of OTC products relevant to oral problems that can be used in a home setting and that may produce positive effects from a preventive, therapeutic or cosmetic point of view.

The present special issue of the Brazilian Oral Research journal (BOR) was dedicated to the proceedings of this symposium. The format of the symposium was based on presentations, in which each presenter was asked to review the literature within a certain field. Each session was followed by a structured discussion. At the end of the symposium a general discussion was also organized. A short summary of the discussions is presented at the end of this issue.

The purpose of this special issue of the BOR is to make the knowledge generated at the symposium available to as broad an audience as possible.

In the first article of the issue, the Public Oral Health (POH) System in Brazil is reviewed. Among the topics covered by it are the different health strategies used since the middle of the last century. In 1951, the SESP (Serviço Especial de Saúde Pública - Special Service of Public Health) implemented the first Oral Health Program. This program was restricted to the schoolchildren population. However, in the 1980's a profound transformation of the health policy at the national level took 
place. As a result, the SUDS (Sistema Unificado e Descentralizado de Saúde - Unified and Decentralized Health System) and later on the SUS (Sistema Único de Saúde - Unified Health System) were established. This new policy was based on the principles of universal attention, decentralization and social control. One milestone in the improvement of the Oral health attention provided by the government was the coverage of collective procedures such as oral hygiene instruction and fluoride mouthrinses. In 1994, the PSF (Programa de Saúde da Família Family Health Care Program) was established. The PSF has been advertised as the main strategy for reorganizing Brazil's new National model for health care. It is based on domiciliary visits, on integrality of care and teamwork. In addition to improving the PSF, the national policy of Oral Health ("Smiling Brazil”) implemented in 2004 promoted an expansion and upgrade of the secondary and tertiary care through the creation of the Center of Dental Specialists (CEO - Centro de Especialidades Odontológicas). Water fluoridation has been an important national strategy in the control of dental caries since the 70's. In the following pages, Pucca Junior et al. ${ }^{1}$ discuss the recent improvements in the National Health policy. Although governmental spending on oral health has increased, it is still inadequate for the demands of the public. Governments throughout the world see OTC products as a way of shifting some of the cost of health care onto consumers. According to the FDA (Food and Drug Administration), OTC products could reduce the inequity in the access to the health system by reducing the cost of treatment. Nowadays, there is a great variety of home-use oral care products available in the marketplace, and an increase in the consumption of these products has been reported in the last decades. Jardim et al. ${ }^{2}$ review the literature on the history and the current global market of oral home-care products.

Dental biofilm is a complex community of microorganisms found on the tooth surface embedded in a matrix of polymers of bacterial and salivary origin. This biofilm is associated with caries, gingivitis and periodontal disease. There are many strategies for managing biofilm. The most common home-care products are the toothbrush (manual or powered) and dental floss. In terms of expenditure, toothbrushes and toothpastes rank first in public spending on oral hygiene products. Many OTC toothpastes include fluoride and other chemicals that may be beneficial in reducing dental caries. The paper by Cury and Tenuta ${ }^{3}$ reviews some concepts of dental caries, the natural phenomenon of enamel remineralization and the effect of fluoride and other remineralizing products. The chemicals present in OTC products could act as antimicrobial agents (controlling cariogenic bacteria) or by enhancing enamel/dentine mineralization. Wolff and Larson ${ }^{4}$ review various aspects of mechanical and chemical cariogenic biofilm control and remineralizing therapeutic approaches. Among the topics covered are the anticariogenic potential of antimicrobials as zinc, triclosan and chlorhexidine, and the remineralizing properties of amorphous calcium phosphate, arginine bicarbonate/calcium carbonate.

The role of dental biofilm as the etiological agent of gingivitis is well documented, and the mechanical control of plaque is clearly the basis of periodontal therapy. However, the difficulty in achieving a proper mechanical plaque control has led researchers to seek chemical antimicrobial agents that could help inhibit biofilm formation on tooth surfaces. Chemical anti-plaque agents could also reach soft tissue surfaces, which serve as a source of bacteria for the colonization of tooth surfaces, thus delaying microbial accumulation on teeth. Toothpastes, in addition to their cleaning potential by way of detergents and abrasives, are vehicles for antimicrobial agents that may have a preventive/therapeutic role in periodontal disease. Mouthrinses are also very popular oral hygiene products with mouth-freshening properties that have the ability of controlling biofilm formation on tooth surfaces. Recent data indicate that home-care products containing chemical antimicrobials can reduce gingivitis beyond what can be accomplished with brushing and flossing. In the article by Teles and Teles ${ }^{5}$, the antimicrobial agents present in formulations of self-care oral hygiene products are fully reviewed. It critically reviews OTC products regarding their anti-plaque and anti-gingivitis effects. In addition, the effects of these chemical agents on the oral microbiota, the long-term safety 
of oral antimicrobial agents and the impact of controlling gingivitis in the development and progression of more severe forms of periodontal diseases are addressed.

Dental erosion is dental wear caused by frequent intake of acidic foods and drinks or due to gastric acid (from reflux or vomiting). There is evidence of a significant increase in dental wear prevalence in deciduous and permanent teeth. Although the presence of acids is a prerequisite for dental erosion, erosive wear is complex and depends on the interaction of biological, chemical and behavioral factors. In order to control mineral loss by erosive wear, it is essential to take into account the multifactorial nature of this event. In the article by Serra et al. ${ }^{6}$ on dental erosion, the etiology, pathophysiology, clinical appearance and effectiveness of self-care measures to control dental erosion are fully reviewed.

For some patients, dentine hypersensitivity is a major problem, causing oral discomfort and pain. It has been described as an acute, provoked pain. It is a very unpleasant situation, limiting the daily eating/drinking habits of individuals. Its prevalence varies greatly among studies (from $4 \%$ to $74 \%$ ). Tooth hypersensitivity is observed when the dentinal tubules of a vital tooth are exposed and subjected to mechanical, chemical and/or thermal stimuli. Common clinical events related to the occurrence of dentine hypersensitivity are gingival recession and tooth wear (erosion/abrasion), exposing dentinal tubules. In addition to the availability of in-office treatment, numerous over-the-counter products are currently available. The mechanisms through which these products may control the pain sensation are the occlusion of dentine tubules and/or nerve stabilization with potassium ions. In the article by Rösing et $a l^{7}$, the mechanisms and factors associated with hypersensitivity and those which may enhance the pain sensation are discussed. The possible treatment

\section{References}

1. Pucca Junior GA, Costa JFR, Chagas LD, Sivestre RM. Oral Health Policies in Brazil. Braz Oral Res. 2009;23(Spec Iss 1):13-20. strategies, with special emphasis on the effects of self-care products for dentine hypersensitivity management, are also covered.

The demand for aesthetic treatment in order to maintain a youthful appearance has increased in the last years. Following this trend, a concern to keep the esthetic appearance of one's smile has grown. Tooth discoloration with age is a reality. With the aging of the population and a decreased prevalence of caries and tooth loss, the demand for tooth whitening products has increased. The traditional tooth bleaching procedure involves the use of custom trays supervised by the dentist. This treatment usually consists in the application of $10 \%$ carbamide peroxide. OTC products appeared as low-cost alternatives to bleach discolored teeth, not requiring dentist supervision. A variety of OTC products such as rinses, paint-on brushes, toothpastes, chewing gums, dental floss, and whitening strips are now available to improve teeth color with low levels of carbamide or hydrogen peroxide. OTC tooth whitening products remove extrinsic stains primarily by means of toothpaste abrasives or by using bleaching products. In the article by Demarco et al. ${ }^{8}$, the current knowledge concerning the efficacy, mechanisms of action and legislation of OTC tooth-bleaching products are discussed at length.

From this introduction, it is clear that over-thecounter products have a great potential to improve oral health. Healthcare is greatly influenced by the resources available. For most countries, governmental spending on healthcare seems insufficient to meet the public needs. Self-care actions, whether by having a healthy life style or by using OTC products, could significantly decrease the cost of healthcare. The trend towards increased self care and a consequent increased empowerment of patients have many potential benefits.

2. Jardim JJ, Alves LS, Maltz M. The history and global market of oral home-care products. Braz Oral Res. 2009;23(Spec Iss $1): 21-6$. 
3. Cury JA, Tenuta LMA. Enamel remineralization: controlling the caries disease or treating early caries lesions? Braz Oral Res. 2009;23(Spec Iss 1):27-34.

4. Wolff MS, Larson C. The cariogenic dental biofilm: good, bad or just something to control? Braz Oral Res. 2009;23(Spec Iss 1):35-42.

5. Teles RP, Teles FRF. Antimicrobial agents used in the control of periodontal biofilms: effective adjuncts to mechanical plaque control? Braz Oral Res 2009;23(Spec Iss 1):43-52.
6. Serra MC, Messias DCF, Turssi CP. Control of erosive tooth wear: possibilities and rationale. Braz Oral Res 2009;23(Spec Iss 1):53-9.

7. Rösing CK, Fiorini T, Liberman DN, Cavagni J. Dentine hypersensitivity: analysis of self-care products. Braz Oral Res 2009;23(Spec Iss 1):60-7.

8. Demarco FF, Meireles SS, Masotti AS. Over-the-counter whitening agents: a concise review. Braz Oral Res 2009;23(Spec Iss 1):68-74. 